\title{
Variación estacional del contenido de betalaína en betarraga (Beta vulgaris L.) cultivada en condiciones de salinidad en el valle de Lluta, Norte de Chile
}

\author{
Seasonal variation of betalaine content in beetroot (Beta vulgaris $L$.) \\ cultivated under salinity conditions in Lluta valley, Northern Chile
}

\author{
Fabiola Astorga ${ }^{1}$, Nancy Luna ${ }^{1}$, Gisell Gómez $z^{1}$, Richard Bustos ${ }^{1}$, \\ Patricia Pacheco ${ }^{1}$, Wladimir Esteban ${ }^{1}$,Yeny Angel ${ }^{1}$, Elizabeth Bastías ${ }^{1 *}$
}

\begin{abstract}
RESUMEN
La betarraga (Beta vulgaris L.) es reconocida por su composición de moléculas bioactivas como la betalaína, que hacen que su consumo aporte importantes beneficios para la salud del ser humano. Su síntesis estaría influenciada por factores como la salinidad, uno de los principales estreses que caracterizan al valle costero desértico de Lluta, ubicado en el extremo norte de Chile, y que junto con el exceso de boro limitan la diversificación agrícola a cultivos tolerantes a esa condición ambiental. En este estudio se evaluó el efecto de la salinidad y la temperatura estacional en la síntesis de betalaína (betacianina y betaxantina) en las variedades Detroit $\left(\mathrm{T}_{0}\right)$ y Larka $\left(\mathrm{T}_{1}\right)$. Se observó que existen diferencias estadísticas significativas en la síntesis de ambas biomoléculas entre las variedades, no así entre las temporadas de cultivo. Los resultados, en cuanto a la síntesis y contenido de betalaína, dan cuenta de la factibilidad de incorporar a Larka como una alternativa productiva para el valle de Lluta, y destinar su producción al consumo fresco o como materia prima para la industria dedicada a la extracción de pigmentos naturales.
\end{abstract}

Palabras clave: pigmento, antioxidante, estrés.

\begin{abstract}
Beetroot (Beta vulgaris $L$.) is recognized for its composition of bioactive molecules such as betalaine, which makes its consumption significantly beneficial for human health. Its synthesis would be influenced by factors such as salinity, one of the main stresses that characterize the coastal desert valley of Lluta, located in the northern of Chile, added to these characteristics is the excess of boron that limits agricultural diversification of the valley to crops tolerant to this environmental condition. In the present study, the effect of salinity and seasonal temperature on the synthesis of betalaine (betacyanin and betaxanthin) in the Detroit $\left(T_{0}\right)$ and Larka $\left(T_{1}\right)$ varieties were evaluated, there are significant statistical differences in the synthesis of both biomolecules amongst varieties, not so between growing seasons. The results, as regards the synthesis and content of betalaine, show the feasibility of incorporating Larka as a productive alternative for the Lluta Valley, being possible to allocate its production to fresh consumption or as a raw material for the industry dedicated to the extraction of natural pigments.
\end{abstract}

Keywords: pigment, antioxidant, stress.

\section{Introducción}

Entre las adaptaciones que suelen presentar algunas plantas en respuesta al desarrollo en condiciones de estrés salino, se encuentra la síntesis de sustancias protectoras o moléculas bioactivas (Stagnari et al., 2014) como la betalaína contenida en miembros del orden Caryophyllales (Cai et al., 2005), al cual pertenece la betarraga
(Beta vulgaris L.). El cultivo de esta planta en los últimos años ha llamado la atención científica internacional al ser considerada un alimento con características funcionales benéficas para la salud del ser humano (Clifford et al., 2015) y una potencial fuente de minerales, antioxidantes, azúcar, fibra dietética, vitaminas, ácidos grasos y biomoléculas con alguna actividad biológica, anticancerígena y radioprotectora (Escribano

\footnotetext{
1 Universidad de Tarapacá, Facultad de Ciencias Agronómicas, Departamento de Producción Agrícola. Arica, Chile.

* Autor por correspondencia: ebastias@uta.cl
} 
et al., 1998). Dentro de su composición se ha identificado la betaxantina, que exhibe una coloración amarillo-naranja y contiene diferentes cadenas laterales de aminoácidos o aminas. En este grupo se incluyen la indicaxantina y vulgaxantinas I y II. Por otra parte están las betacianinas, de coloración rojo-violeta, que contienen un residuo de ciclo-dihidro xifenilalanina (ciclo-DOPA) e incluyen la betanina, isobetanina y neobetanina (Chhikara et al., 2019). Todos estos compuestos son reconocibles comercialmente como colorantes alimentarios debido a su naturaleza no precaria, no tóxica y no cancerígena.

Su síntesis estaría influenciada por factores como la variedad (Niveyro et al., 2013), estado de maduración (Castellar et al., 2012), incremento de la temperatura (Szopínska y Gaweda, 2013), salinidad (Mulry et al., 2015), luz, nutrientes, reguladores de crecimiento (Bhuiyan et al., 2002) y disponibilidad de agua (Stagnari et al., 2014). En la actualidad existen muy pocos estudios conducentes a evaluar los efectos de los estreses abióticos en la producción y acumulación de esas biomoléculas en este cultivo. En tal sentido, el objetivo de este trabajo fue evaluar la acumulación de ese pigmento en las raíces de betarraga, en dos estaciones del año, en condiciones de salinidad y temperatura del valle de Lluta, ubicado en el norte de Chile, surgiendo como una posibilidad de diversificación productiva para esta zona salina.

\section{Materiales y métodos}

\section{Sitio del experimento}

El cultivo se realizó al aire libre durante dos estaciones del año (temporada 1: cosecha de frutos hecha en el verano del 2013 (91 Días Después de Trasplante (DDT) y temporada 2: cosecha de frutos en invierno-primavera del año 2014 (99 DDT)) en la parcela experimental de la Facultad de Ciencias Agronómicas de la Universidad de Tarapacá, km 19, sector Rosario del valle de Lluta. Las características químicas del agua de riego y suelo se presentan en las Tablas 1 y 2.

El material vegetal utilizado para la cuantificación de betalaína incluyó la variedad Detroit $\left(\mathbf{T}_{\mathbf{0}}\right)$ de la empresa Bonanza Seeds que es la más cultivada en el valle de Lluta. Se caracteriza por su forma aglobada y piel lisa, así como por su alta uniformidad y productividad. Puede sembrarse durante todo el año y tiene maduración comercial a los 60 días. El segundo tratamiento $\left(\mathbf{T}_{1}\right)$ correspondió a la variedad Larka de la empresa Semillas Latinoamericanas, una betarraga multigérmica, de precocidad media y follaje vigoroso. Presenta raíces muy homogéneas, redondas y de color rojo intenso. Es recomendada para cultivos de verano y otoño.

Se realizó siembra directa en el terreno previamente preparado con una mezcla de arena y guano de cordero, utilizando un marco de plantación de 0,2 x 1,2 m (41.666 plantas ha ${ }^{-1}$ ) y un largo de camellón de $27 \mathrm{~m}$. Durante su desarrollo el cultivo fue regado por goteo y fertilizado según sus requerimientos.

El registro meteorológico de las variables temperatura $\left({ }^{\circ} \mathrm{C}\right)$ y humedad relativa $(\%)$ de los meses de estudio (Tabla 3) se obtuvo de las estaciones en línea de la Red Agrometeorológica (AGROMET) del Instituto de Investigaciones Agropecuarias (INIA).

\section{Diseño experimental y tratamientos:}

El experimento se realizó con un diseño de bloques completos al azar, con 2 tratamientos $\left(\mathrm{T}_{0}\right.$ : Detroit y $\mathrm{T}_{1}$ : Larka), 4 repeticiones y una superficie total de $259,2 \mathrm{~m}^{2}$ por tratamiento.

La cuantificación de betalaína se hizo en 3 etapas: pre-tratamiento de la muestra, preparación de muestras con $\mathrm{N}_{2}$ líquido y liofilización.

Pre-tratamiento de la muestra: Se colectaron4 muestras homogéneas de cada bloque, de ambas variedades, seleccionando la cuarta parte de cada fruto del bloque (incluida la cáscara) para ser lavadas, ralladas y almacenadas en bolsas herméticas refrigeradas a $5{ }^{\circ} \mathrm{C}$.

Tratamiento con nitrógeno líquido: En un mortero se incorporó una pequeña cantidad de betarraga rallada a la que se agregaron pequeñas dosis de nitrógeno líquido hasta cubrir por completo la muestra para su trituración, pulverización y posterior depósito en tubos falcon de $50 \mathrm{ml}$, mantenidos en un cooler con hielo. El material congelado se introdujo en una cámara de vacío (liofilizador modelo BK-FD10P, Biobase Biodustry (Shandong) Co., Ltd.) para realizar la separación del agua de las muestras por sublimación. Para acelerar el proceso se utilizaron ciclos de congelación-sublimación y se consiguió eliminar la totalidad del agua libre contenida. Para la 
Tabla 1. Características químicas promedios de agua de riego, Temporada 1 y Temporada 2. Sector Rosario, km 19, valle de Lluta.

\begin{tabular}{lclcc}
\hline Parámetro & Unidad & Método & Temporada 1 & Temporada 2 \\
\hline Ce (Extracto Sat.) & $\mathrm{mS} \mathrm{cm}^{-1}$ & Conductimetría & 2,44 & 2,76 \\
pH (Agua) & - & Potenciométrico & 7,16 & 7,68 \\
Calcio $\left(\mathrm{Ca}^{2+}\right.$ & $\mathrm{mg} \mathrm{L}$ & Extracto Sat. & 185,14 & 282 \\
Magnesio $\left(\mathrm{Mg}^{2+}\right)$ & $\mathrm{mg} \mathrm{L}^{-1}$ & Extracto Sat. & 39,14 & 38,64 \\
Potasio $\left(\mathrm{K}^{+}\right)$ & $\mathrm{mg} \mathrm{L}^{-1}$ & Extracto Sat. & 32,8 & 29,5 \\
Sodio $\left(\mathrm{Na}^{+}\right)$ & $\mathrm{mg} \mathrm{L}^{-1}$ & Extracto Sat. & 250,2 & 215,72 \\
Cloruros $\left(\mathrm{Cl}^{-}\right)$ & $\mathrm{mg} \mathrm{L}^{-1}$ & Extracto Sat. & 489,6 & 511,2 \\
Sulfato $\left(\mathrm{SO}_{4}^{2-}\right)$ & $\mathrm{mg} \mathrm{L}^{-1}$ & Extracto Sat. & 379,15 & 426 \\
Nitrato $\left(\mathrm{NO}_{3}^{-}\right)$ & $\mathrm{mg} \mathrm{L}^{-1}$ & Extracto Sat. & 3,86 & 2,42 \\
Bicarbonato $\left(\mathrm{HCO}_{3}^{-}\right)$ & $\mathrm{mg} \mathrm{L}^{-1}$ & Extracto Sat. & 73,2 & 183 \\
Carbonato $\left(\mathrm{CO}_{3}^{2-}\right)$ & $\mathrm{mg} \mathrm{L}^{-1}$ & Extracto Sat. & $\mathrm{ND}$ & $\mathrm{ND}$ \\
Boro (B) & $\mathrm{mg} \mathrm{L}^{-1}$ & Extracto Sat. & 10,85 & 14,5 \\
\hline
\end{tabular}

Fuente. Laboratorio de suelo y agua, Facultad de Ciencias Agronómicas, Universidad de Tarapacá. Años 2013 y 2014. *ND: No detectable.

Tabla 2. Características químicas promedios de suelo, Temporada 1 y Temporada 2. Sector Rosario, km 19, valle de Lluta.

\begin{tabular}{lclrr}
\hline Parámetro & Unidad & Método & Temporada 1 & Temporada 2 \\
\hline Ce (Extracto Sat.) & $\mathrm{mS} \mathrm{cm}^{-1}$ & Conductimetría & 27,4 & 107,1 \\
pH (Agua) (Maff 1:2,5) & - & Potenciométrico & 7,2 & 6,3 \\
$\mathrm{~N}-\mathrm{NO}_{3}$ Disponible & $\mathrm{mg} \mathrm{Kg}^{-1}$ & Fenoldisulfónico & 14,6 & 38,7 \\
P-Disponible & $\mathrm{mg} \mathrm{Kg}^{-1}$ & Olsen & 120,5 & 20,8 \\
K-Disponible & $\mathrm{mg} \mathrm{Kg}^{-1}$ & Acetato amonio & 1477,5 & 2012,5 \\
Materia Orgánica & $\%$ & Dicromato sodio & 1,6 & 2,0 \\
Calcio $\left(\mathrm{Ca}^{2+}\right)$ & $\mathrm{mg} \mathrm{L}^{-1}$ & Extracto Sat. & 1297,5 & 3960,5 \\
Magnesio $\left(\mathrm{Mg}^{2+}\right)$ & $\mathrm{mg} \mathrm{L}^{-1}$ & Extracto Sat. & 592,5 & 3045,0 \\
Potasio $\left(\mathrm{K}^{+}\right)$ & $\mathrm{mg} \mathrm{L}^{-1}$ & Extracto Sat. & 412,5 & 912,5 \\
Sodio $\left(\mathrm{Na}^{+}\right)$ & $\mathrm{mg} \mathrm{L}^{-1}$ & Extracto Sat. & 3547,5 & 652,5 \\
Cloruros $\left(\mathrm{Cl}^{-}\right)$ & $\mathrm{mg} \mathrm{L}^{-1}$ & Extracto Sat. & 2978,9 & 43878,0 \\
Sulfato $\left(\mathrm{SO}_{4}^{2-}\right)$ & $\mathrm{mg} \mathrm{L}^{-1}$ & Extracto Sat. & 8287,6 & 1737,0 \\
Nitrato $\left(\mathrm{NO}_{3-}\right)$ & $\mathrm{mg} \mathrm{L}^{-1}$ & Extracto Sat. & 26,2 & 283,4 \\
Bicarbonato $\left(\mathrm{HCO}_{3-}\right)$ & $\mathrm{mg} \mathrm{L}^{-1}$ & Extracto Sat. & 219,6 & 213,5 \\
Carbonato $\left(\mathrm{CO}_{3}{ }^{2-}\right)$ & $\mathrm{mg} \mathrm{L}^{-1}$ & Extracto Sat. & $\mathrm{ND}$ & $\mathrm{ND}$ \\
Boro $(\mathrm{B})$ & $\mathrm{mg} \mathrm{L}^{-1}$ & Extracto Sat. & 13,2 & 51,2 \\
\hline
\end{tabular}

*ND: No detectable.

Fuente. Laboratorio de suelo y agua, Facultad de Ciencias Agronómicas, Universidad de Tarapacá. Años 2013 y 2014.

Tabla 3. Registro meteorológico del sector de estudio.

\begin{tabular}{lccccccc}
\hline & \multicolumn{3}{c}{ Temporada 1 (Verano) } & & \multicolumn{3}{c}{ Temporada 2 (Invierno-Primavera) } \\
\hline \multicolumn{1}{c}{ Mes } & $\begin{array}{c}\text { Temperatura } \\
\text { Mínima }\left({ }^{\circ} \mathrm{C}\right)\end{array}$ & $\begin{array}{c}\text { Temperatura } \\
\text { Máxima }\left({ }^{\circ} \mathrm{C}\right)\end{array}$ & $\begin{array}{c}\text { Humedad } \\
\text { Relativa }(\%)\end{array}$ & Mes & $\begin{array}{c}\text { Temperatura } \\
\text { Mínima }\left({ }^{\circ} \mathrm{C}\right)\end{array}$ & $\begin{array}{c}\text { Temperatura } \\
\text { Máxima }\left({ }^{\circ} \mathrm{C}\right)\end{array}$ & $\begin{array}{c}\text { Humedad } \\
\text { Relativa }(\%)\end{array}$ \\
\hline Octubre 2013 & 9,5 & 25,0 & 70,8 & Mayo 2014 & 7,1 & 23,9 & 71,9 \\
Noviembre 2013 & 7,9 & 27,3 & 67,0 & Junio 2014 & 6,1 & 21,8 & 75,9 \\
Diciembre 2013 & 11,5 & 28,5 & 62,9 & Julio 2014 & 5,0 & 24,2 & 76,6 \\
Enero 2014 & 13,8 & 30,9 & 57,3 & Agosto 2014 & 6,5 & 25,6 & 76,7 \\
Promedio & 10,7 & 27,9 & 64,5 & Promedio & 6,2 & 23,9 & 75,3 \\
\hline
\end{tabular}

Fuente. Red Agrometeorológica (AGROMET), Instituto de Investigaciones Agropecuarias (INIA), 2017. 
cuantificación del contenido total de betalaína se utilizó la metodología indicada por FernándezLópez y Almela (2001) mediante evaluación espectrofotométrica (HPLC-grade).

Extracción de betalaína: Se tomó una muestra de $2 \mathrm{~g}$ de pulpa liofilizada y se colocó en un matraz Erlenmeyer con $20 \mathrm{ml}$ de metanol acuoso $80 \%$ (V/V). La mezcla se sonicó por 10 minutos en un Bath sonicador (Branson model ${ }^{\circledR}$ Danbury, USA) previo a su agitación por 20 minutos en un agitador horizontal (modelo G10 R, New Jersey, USA) a temperatura ambiente y en oscuridad. Cada muestra se centrifugó a 2200 x g por 10 minutos en una centrífuga (Hettich Zentrifugen ${ }^{\circledR}$ Mod. Universal 32, Germany). El sobrenadante se guardó y el residuo se sometió a una segunda extracción con la metodología descrita anteriormente. Los sobrenadantes se juntaron y se filtraron con papel Whatman Número 4, para luego concentrarlos a sequedad en un rotavapor (Buchi ${ }^{\circledR}$ Modelo R-125, Switzerland) a $40^{\circ} \mathrm{C}$.

Cuantificación: La cuantificación de betacianinas y betaxantinas se realizó según lo descrito por Castellanos-Santiago y Yahia (2008), mediante la absorbancia de los extractos de las betalaínas a 538 y $483 \mathrm{~nm}$, respectivamente, en un espectrofotómetro (Perkin Elmer Lambda $25^{\circledR} \mathrm{UV} /$ Vis, (USA)). Para la conversión de las unidades de absorbancia en unidades de concentración se utilizó la expresión: B $(\mathrm{mg} / \mathrm{g})=(\mathrm{A} \times \mathrm{FD} \times \mathrm{PM} \times \mathrm{V}) /(\varepsilon \times$ $\mathrm{P} \times \mathrm{L}$ ), donde $\mathrm{B}$ es betacianina o betaxantina, $\mathrm{A}$ es la absorbancia $538 \mathrm{~nm}$ para betacianina y 483 $\mathrm{nm}$ para betaxantina, FD es el factor de dilución al momento de leer en el espectofotómetro, PM es el peso molecular $($ Betanina $=550 \mathrm{~g} / \mathrm{mol}$ e Indicatina $=308 \mathrm{~g} / \mathrm{mol}$ ), $\mathrm{V}$ es el volumen del extracto, $\varepsilon$ es el peso molecular $($ Betanina $=60.000 \mathrm{~L} / \mathrm{mol} \mathrm{cm}$, e Indicatina $=48.000 \mathrm{~L} / \mathrm{mol} \mathrm{cm}$ ) y L es la longitud de la celda $(1 \mathrm{~cm})$. Considerando lo anterior, se realizó el cálculo mediante la expresión matemática:

$\mathrm{CB}(\mathrm{mg} / \mathrm{g} \cdot \mathrm{ps})=\left(\mathrm{A}^{*} \mathrm{FD}^{*} \mathrm{MW}+\mathrm{V}\right) / \mathrm{e}^{*}$ peso seco $)$

Donde:

$\mathrm{CB}=$ Contenido de betalaína

$\mathrm{A}=$ Absorbancia

$\mathrm{F}=$ Factor peso molecular

$\mathrm{V}=$ Volumen total

$\mathrm{E}=$ Coeficiente de extinción

*Para betacianinas se utilizó una masa de $550 \mathrm{~g} / \mathrm{mol}$ $\mathrm{y}$ un $\mathrm{E}=60.000$, y para betaxantina una masa de
$308 \mathrm{~g} / \mathrm{mol}$ y un $\mathrm{E}=48.000$ (Castellanos-Santiago y Yahia, 2008).

\section{Análisis de datos}

Se utilizó el programa estadístico SPSS versión 22/PC (2013) y la prueba T de Student para dos pruebas independientes con $\mathrm{P} \leq 0,05(95 \%)$.

\section{Resultados}

\section{Contenido de betacianina}

Durante la temporada 1 (Tabla 4) se presentaron diferencias significativas $(P \leq 0,05)$ en la síntesis de betacianina entre $\mathrm{T}_{0}\left(1,41 \mathrm{mg} \mathrm{g}^{-1} \mathrm{PS}\right)$ y $\mathrm{T}_{1}$ (3,38 $\left.\mathrm{mg} \mathrm{g}^{-1} \mathrm{PS}\right)$, con resultados similares durante la temporada 2 (1,32 $\mathrm{mg} \mathrm{g}^{-1} \mathrm{PS}$ en Detroit y $3,32 \mathrm{mg}$ $\mathrm{g}^{-1}$ PS en Larka). Se observaron diferencias estadísticas en la síntesis del pigmento entre ambas variedades. Al comparar el contenido de betacianina en $\mathrm{T}_{0}$, entre temporadas, no se detectaron diferencias significativas en cuanto a la síntesis de esta biomolécula, comportamiento que se observó notoriamente también en $\mathrm{T}_{1}$.

\section{Contenido de betaxantina}

Los resultados observados durante la temporada 1 mostraron diferencias significativas $(\mathrm{P} \leq 0,05)$ en cuanto a la síntesis de betaxantina entre $\mathrm{T}_{0} \mathrm{y}$ $\mathrm{T}_{1}$ (3,34 y 6,31 $\mathrm{mg} \mathrm{g}^{-1} \mathrm{PS}$, respectivamente). Esta tendencia se repitió durante la temporada $2(3,43$ y 6,63 $\mathrm{mg} \mathrm{g}^{-1} \mathrm{PS}$, respectivamente).

Al comparar el contenido de betaxantina en $\mathrm{T}_{0}$, entre ambas temporadas $\left(3,34\right.$ y $3,43 \mathrm{mg} \mathrm{g}^{-1}$ PS, respectivamente), no se detectaron diferencias estadísticas significativas en cuanto a su síntesis, comportamiento similar al observado en $\mathrm{T}_{1}(6,31$ y 6,63 $\mathrm{mg} \mathrm{g}^{-1} \mathrm{PS}$, respectivamente).

Por otra parte, en ambas temporadas se observó un mayor contenido de betaxantinas, respecto a betacianinas, en los dos tratamientos (Tabla 4), y hubo diferencias significativas en la síntesis entre ambos pigmentos.

\section{Rendimiento de frutos}

Los rendimientos en la temporada 1 mostraron diferencias significativas entre $\mathrm{T}_{0}$ y $\mathrm{T}_{1}(15,1$ y 9,42 Ton $\mathrm{ha}^{-1}$, respectivamente), tendencia similar a la 
Tabla 4. Síntesis de betacianina y betaxantina, Temporada 1 y Temporada 2. Sector Rosario, km 19, valle de Lluta.

\begin{tabular}{|c|c|c|c|c|c|c|}
\hline \multicolumn{7}{|c|}{ Temporada 1 (Verano) } \\
\hline \multirow[b]{2}{*}{ Variedad } & \multicolumn{3}{|c|}{ Betacianina } & \multicolumn{3}{|c|}{ Betaxantina } \\
\hline & $\mathrm{mg} \mathrm{g}^{-1} \mathrm{PS}$ & $\mathrm{MS}(\mathrm{g} / \mathrm{Ha})$ & $\begin{array}{l}\text { Contenido por superficie } \\
\qquad(\mathrm{g} / \mathrm{Ha})\end{array}$ & $\mathrm{mg} \mathrm{g}^{-1} \mathrm{PS}$ & MS (g/Ha) & $\begin{array}{l}\text { Contenido por superficie } \\
\qquad(\mathrm{g} / \mathrm{Ha})\end{array}$ \\
\hline Detroit $\left(\mathrm{T}_{0}\right)$ & $1,41 \pm 0,05$ & 1.077 .139 & 1.519 & $3,34 \pm 0,85$ & 1.077 .139 & 3.598 \\
\hline Larka $\left(\mathrm{T}_{1}\right)$ & $3,38 \pm 0,81$ & 672.445 & 2.273 & $6,31 \pm 2,01$ & 672.445 & 4.243 \\
\hline \multicolumn{7}{|c|}{ Temporada 2 (Invierno-Primavera) } \\
\hline & \multicolumn{3}{|c|}{ Betacianina } & \multicolumn{3}{|c|}{ Betaxantina } \\
\hline Variedad & $\mathrm{mg} \mathrm{g}^{-1} \mathrm{PS}$ & MS (g/Ha) & $\begin{array}{c}\text { Contenido por superficie } \\
\text { (g/Ha) }\end{array}$ & $\mathrm{mg} \mathrm{g}^{-1} \mathrm{PS}$ & MS (g/Ha) & $\begin{array}{c}\text { Contenido por superficie } \\
\qquad(\mathrm{g} / \mathrm{Ha})\end{array}$ \\
\hline Detroit $\left(\mathrm{T}_{0}\right)$ & $1,32 \pm 0,13$ & 2.230 .725 & 2.945 & $3,43 \pm 0,87$ & 2.230 .725 & 7.651 \\
\hline Larka $\left(\mathrm{T}_{1}\right)$ & $3,32 \pm 0.83$ & 1.806 .210 & 5.997 & $6,63 \pm 2,32$ & 1.806 .210 & 11.975 \\
\hline
\end{tabular}

(PS) : Peso seco.

(*) : Significativo con $\mathrm{p} \leq 0,05$.

(MS): Materia seca.

registrada durante la temporada $2(21,2$ y 17,20 Ton $\mathrm{ha}^{-1}$, respectivamente). $\mathrm{T}_{0}$ fue el tratamiento que presentó en ambas temporadas el mayor rendimiento. Respecto al rendimiento de cada tratamiento, entre las temporadas 1 y 2 , los resultados mostraron la existencia de diferencias estadísticas en el de $\mathrm{T}_{0}$ y $\mathrm{T}_{1}$, y fueron significativamente mayores los rendimientos durante la temporada 2 (inviernoprimavera), tanto en $\mathrm{T}_{0}$ como en $\mathrm{T}_{1}$ (Figura 1).

\section{Discusión}

Según Lee y Wiley (1981), la temperatura sería uno de los factores que tendrían un rol importante en la síntesis de las betalaínas durante el desarrollo

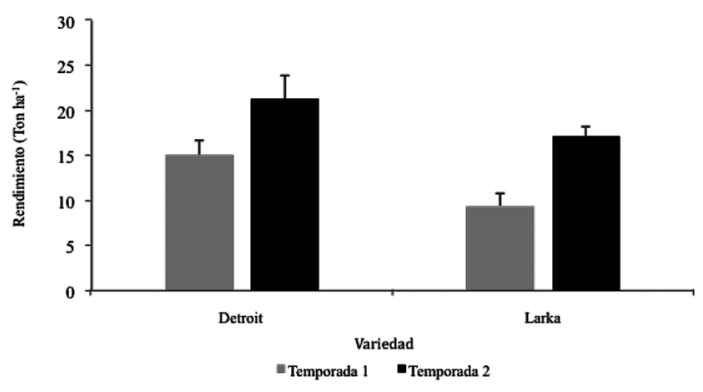

Figura 1. Rendimiento de betarraga (Beta vulgaris $\mathrm{L}$ ) variedades Detroit $\left(\mathrm{T}_{0}\right)$ y Larka $\left(\mathrm{T}_{1}\right)$, Temporada 1 y Temporada 2 . Sector Rosario, km 19, valle de Lluta. del cultivo. Sin embargo, los resultados obtenidos indicarían que el cambio de temporada, y con esto, la fluctuación de las temperaturas ambientales registradas durante los períodos evaluados (Tabla 3), no tendrían un efecto en la síntesis de este pigmento en $\mathrm{T}_{0}$ y $\mathrm{T}_{1}$, o tal vez no la afectarían en condiciones de campo.

Otros autores mencionan que la acumulación de betalaína podría estar influenciada por diversos factores, tanto bióticos como abióticos, y relacionarse con la variedad (Niveyro et al., 2013), estado de madurez (Castellar et al., 2012) y la concentración de micronutrientes en la planta (Tyszka-Czochara et al., 2016). Entre los factores ambientales, el estrés salino sería uno de los principales precursores en la síntesis de este tipo de pigmento en respuesta al exceso de sales presentes en el medio (Jain y Gould, 2015a), y que caracteriza los suelos y el agua de riego del valle de Lluta (Bastías et al., 2004). Al observar los análisis de ambas temporadas, tanto en agua como en suelo (Tabla 1 y 2), se evidencian altos y variables contenidos de sales con exceso de $\mathrm{Na}^{+}$, $\mathrm{Cl}^{-}$y $\mathrm{B}$ que podrían inducir alguna respuesta fisiológica en cultivos poco tolerantes. Es el caso de $\mathrm{T}_{1}$, que sintetizó un mayor contenido de betacianina y betaxantina, en ambas temporadas, siendo tal vez una variedad menos sensible a este tipo de estrés, respecto a $\mathrm{T}_{0}$. El comportamiento 
de $\mathrm{T}_{1}$ coincidiría con el de las halófitas Disphyma australe, Suaeda salsa y Portulaca oleracea, que presentarían una mayor acumulación de betalaína en presencia de estrés salino (Jain y Gould, 2015b; Wang et al., 2006; Mulry et al., 2015).

Se destaca la diferencia entre los niveles de pigmentos sintetizados, duplicándose el contenido de betaxantina en ambas temporadas, tanto en $\mathrm{T}_{0}$ como en $\mathrm{T}_{1}$ (Tabla 4). Este último tratamiento (cv. Larka) fue el que acumuló un mayor contenido de pigmento en ambas temporadas. Esta mayor acumulación de betaxantina coincidiría con los resultados reportados por Valencia et al. (2017) en semillas de quinoa peruana.

Esta escasa documentación de menor o mayor síntesis de uno u otro pigmento y su funcionalidad en situaciones de estrés y, particularmente, en condiciones de salinidad, nos permite sugerir la importancia de realizar estos trabajos considerando el comportamiento de esta biomolécula bajo estrés abiótico.

Se sugiere que factores como la salinidad y el efecto varietal u otros, aún no evaluados, como los mencionados por Kujala et al. (2002) y Belhadj et al. (2017), podrían ser los reguladores o precursores en la síntesis de estas biomoléculas.

Respecto a los rendimientos, se pudo observar un mejor comportamiento de ambas variedades de betarragas durante la temporada de invierno-primavera. Se registraron resultados estadísticos más favorables en la variedad Detroit $\left(\mathrm{T}_{0}\right)$, debido posiblemente a las mejores características del agua de riego y las temperaturas más adecuadas que explicarían el mayor desarrollo y rendimiento del cultivo.

Si bien es cierto que $\mathrm{T}_{0}$ alcanzó mayores rendimientos que $\mathrm{T}_{1}$, esta diferencia se compensaría en $\mathrm{T}_{1}$ por la mayor concentración de betalaína (betacianina y betaxantina $(\mathrm{g} / \mathrm{Ha})$ ) en las raíces de betarraga (Tabla 4), lo cual en definitiva es el interés de la industria dedicada a la extracción de pigmentos, con un ahorro de insumos y agua.

\section{Conclusión}

La síntesis de betalaína en ambas variedades de betarraga no tendría una relación directa con la estacionalidad y la variación de temperaturas durante el desarrollo del cultivo. Existe, más bien, un efecto de la salinidad y de la variedad en la síntesis y acumulación de esta biomolécula en las raíces de Detroit y mayormente en Larka.

La temporada 2 (invierno primavera) favoreció el rendimiento de ambas variedades de betarraga y en especial el de Detroit, que fue mayor. No obstante, el contenido de betalaína por superficie fue mayor en Larka, en ambas temporadas. Por ello resulta interesante analizar si el destino de la producción de betarraga se orienta a la industria de la extracción y producción de pigmentos como materia prima.

Cabe destacar que la síntesis de estos pigmentos en betarraga cultivada en condiciones de campo y su respuesta a estrés abiótico (alta radiación, temperatura, salinidad y estrés hídrico) ha sido escasamente documentada. Se abre así un campo interesante para estudios que orienten respecto del incremento de la producción de estas biomoléculas en cultivos desarrollados en suelos marginales.

\section{Agradecimientos}

Proyecto Convenio de Desempeño UTA 1795. "Fortalecimiento de la investigación interdisciplinaria en los recursos genéticos agrícolas ancestrales y aumento de su valorización en los custodios de la Región de Arica y Parinacota" y el Laboratorio de Fisiología y Biotecnología Vegetal, Universidad de Santiago de Chile (USACH).

\section{Literatura Citada}

Bastías, E.; González-Moro, M.; González-Murua, C. 2004. Zea mays L. amylacea from the Lluta Valley (AricaChile) tolerates salinity stress when high levels of boron are available. Plant and Soil 267: 73-84.

Belhadj Slimen, I.; Najar, T.; Abderrabba, M.

2017. Chemical and antioxidant properties of betalains. $J$. Agric. Food Chem. 65: 675-689.

Bhuiyan, N.; Murakami, K.; Adachi, T.

2002. Variation in betalain content and factors affecting the biosynthesis in Portulaca sp. "Jewel" cell cultures. Plant Biotechnology 19 (5): 369-376.
Cai, Y.; Sun, M.; Corke, H.

2005. Characterization and application of betalain pigments from plants of the Amaranthaceae. Trends in Foods Science \& Technology 16: 370-376.

Castellanos-Santiago, E.; Yahia, E.

2008. Identification and quantification of betalains from the fruits of 10 mexican prickly pear cultivars by highperformance liquid chromatography and electrospray ionization mass spectrometry. J. Agric. Food Chem. 56: 5758-5764. 
Castellar, M.; Solano, F.; Obón, J.

2012. Betacyanin and other antioxidants production during growth of Opuntia stricta (Haw.) fruits. Plant Foods Hum Nutr. 67: 337-343.

Chhikara, N.; Kushwaha, K.; Sharma, P.; Gat, Y.; Panghal, A. 2019. Bioactive compounds of beetroot and utilization in food processing industry. A critical review. Food Chemistry 272: 192-200.

Clifford, T.; Howatson, G.; Daniel, J.; West, D. J.; Stevenson, E. J. 2015. The potential benefits of red beetroot supplementation in health and disease. Nutrients, 7: 2801-2822.

Escribano, J.; Pedreño, M.; García-Carmona, F.; Muñoz, R. 1998. Characterization of the antiradical activity of betalains from Beta vulgaris L. roots. Phytochemical Analysis 9: 124-127.

Fernández-López, J.; Almela, L.

2001. Application of high-performance liquid chromatography to the characterization of the betalain pigments in prickly pear fruits. Journal of Chromatography A. 913: 415-420.

Jain, G.; Gould, K.

2015a. Are betalain pigments the functional homologues of anthocyanins in plants? Environmental and Experimental Botany 119: 48-53.

Jain, G.; Gould, K.

2015b. Functional significance of betalain biosynthesis in leaves of Disphyma australe under salinity stress. Environmental and Experimental Botany 109: 131-140.

Kujala, T.; Vienola, M.; Klika, K.

2002. Betalain and phenolic compositions of four beetroot (Beta vulgaris) cultivars. Eur Food Technol 214: 505-510.

Lee, Y.; Wiley, C.

1981. Betalaine yield from a continuous solid-liquid extraction system as influenced by raw product, post-harvest and processing variables. Journal of Food Science 46: 422-424.
Mulry, K.; Hanson, B.; Dudle, D.

2015. Alternative strategies in responde to saline stress in two varieties of Portulaca oleracea (Purslane). Two Varieties of Portulaca oleracea (Purslane). PLoS ONE 10(9): e0138723. doi: 10.1371/journal. pone.0138723.

Niveyro, S.L.; Mortensen, A.G.; Fomsgaard, I.S.; Salvo, A. 2013. Differences among five amaranth varieties (Amaranthus spp.) regarding secondary metabolites and foliar herbivory by chewing insects in the field. Arthropod-Plant Interactions 7: 235-245.

Stagnari, F.; Galieni, A.; Speca, S.; Pisante, M.

2014. Water stress effects on growth, yield and quality traits of red beet. Scientia Horticulturae 165: 13-22.

Szopinska, A.; Gaweda, M.

2013. Comparison of yield and quality of red beet roots cultivated using conventional, integrated and organic method. Journal of Horticultural Research 21 (1): 107-114.

Tyszka-Czochara, M.; Pasko, P.; Zagrodzki, P.; Gajdzik, E.;

Wietecha-Posluszny, R.; Gorinstein, S.

2016. Selenium supplementation of amaranth sprouts influences betacyanin content and improves anti-inflammatory properties via NFKB in murine RAW 264.7 macrophages. Biological Trace Element Research, 169: 320-330.

Valencia, Z.; Cámara, F.; Ccapa, K.; Catacora, P.; Quispe, F. 2017. Compuestos bioactivos y actividad antioxidante de semillas de quinua peruana (Chenopodium quinoa W.). Revista Sociedad Química de Perú 83 (1): 16-29.

Wang, C.; Zhao, J.; Chen, M.; Wang, B.

2006. Identification of betacyanin and effects of environmental factors on its accumulation in halophyte Suaeda salsa L. J. Plant. Physiol. Mol. Biol., 32: 195-201. 
